\title{
Leukocytoclastic vasculitis and polyarthralgia in scrub typhus: An unusual presentation
}

\section{Arinaganahalli Subbanna \\ Praveen Kumar, Anupama \\ Monohar Prasad", Lakshmi Gumpeny, Revena Siddappa}

Department of Medicine, PES Institute of Medical Science and Research, Kuppam, Chittor District,

Andhra Pradesh, ${ }^{1}$ Department

of Dermatology, Madras Medical College, Chennai, Tamil Nadu, India

Address for the Correspondence:

Dr. A. S. Praveen Kumar, Department of Medicine, PES Institute of Medical Science and Research, Kuppam - 517 425,

Chittor District,

Andhra Pradesh, India.

E-mail: jipmer.praveen@ gmail.com

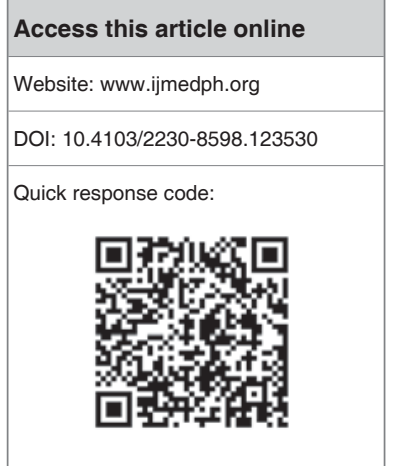

Scrub typhus is an acute, febrile zoonosis caused by an obligate intracellular bacterium Orientia tsutsugamushi. It is of greatest public health importance in tropical rural Asia. The clinical manifestations of the disease range from sub-clinical to fatal organ failure. The common symptoms are fever, chills, headache, myalgia, dry cough, lymphadenopathy and gastrointestinal disturbances. The first presentation with cutaneous vasculitis and polyarthralgia is rare and unusual. We report a case of scrub typhus that presented with cutaneous vasculitis and polyarthralgia, which is very unusual and patient recovered with doxycycline treatment.

Key words: Chigger, eschar, leukocytoclastic vasculitis, Orientia tsutsugamushi, polyarthralgia, Weil-Felix test

\section{INTRODUCTION}

Scrub typhus is an acute febrile illness caused by Orientia tsutsugamushi, which is transmitted to humans by the bite of a larval-stage trombiculid mite or chigger. It is an important consideration in the differential diagnosis of acute febrile illness, especially from endemic areas. ${ }^{[1]}$ The common symptoms are fever, chills, headache, myalgia, dry cough, lymphadenopathy and gastrointestinal disturbances. ${ }^{[2]}$ Scrub typhus can affect skin, lung, heart and central nervous system with potential of causing serious life-threatening complications. The clinical and laboratory features are non-specific in scrub typhus. The eschar is the single most useful diagnostic clue and is pathognomonic for O. tsutsugamushi, but is seen in less than $10 \%$ of cases in the Indian subcontinent. ${ }^{[3]}$

\section{CASE REPORT}

A 50-year-old housewife presented with 7 days history of fever with chills, headache and with reddish papulomacular lesions predominantly distributed over both lower limbs. She also had a history of multiple joint pains and myalgia. On examination, her pulse rate was $96 / \mathrm{min}$, blood pressure of 130/80 $\mathrm{mmHg}$ and respiratory rate of $20 / \mathrm{min}$. Skin examination revealed multiple palpable erythematous plaques predominantly distributed over both lower limbs [Figure 1]. The lesions are non-blanchable to pressure. The musculoskeletal examination showed calf muscle tenderness and there was no joint swelling. The other system examination was within the normal limits. Laboratory examination revealed normal renal and liver function tests. The peripheral smear and total count was normal. The ELISA for O. tsusgamushi was positive. Skin biopsy done on day 2 of hospital admission (day 8 of fever and rash) revealed leukocytoclastic vasculitis [Figure 2]. The blood and urine cultures were sterile. Leptospira serology and WIDAL were negative.

She was started on doxycycline (100 $\mathrm{mg}$ bid orally). Her arthralgia resolved completely in $48 \mathrm{~h}$ after treatment. The skin lesion took 7 days to resolve completely [Figure 1b] and patient discharged from hospital on day 8.

\section{DISCUSSION}

O. tsutsugamushi is an obligate intracellular bacterium that was recently reclassified from the genus Rickettsia into a separate genus. This is because it differs from Rickettsiae in genetic composition, cell wall structure and multiplication cycle. In the pre-antibiotic era scrub typhus was dreaded 


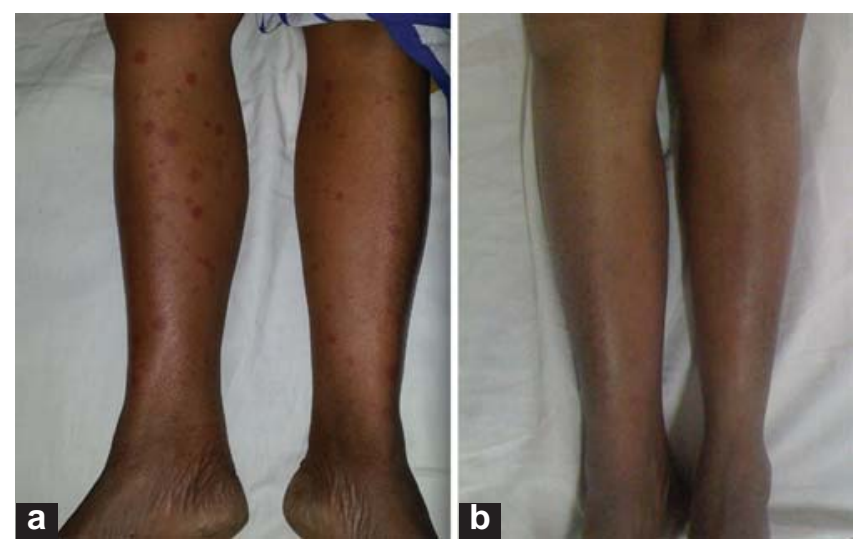

Figure 1: Multiple palpable erythematous plaques distributed over both lower limbs (a) and (b) lesions completely resolved after treatment

disease. The clinical severity of scrub typhus ranges from mild to fatal organ failure. The usual clinical features are headache, high fever and myalgia. A non-pruritic macular or papular rash may be seen on the trunk, which can later spread to the face, arms and legs. The various complications known to occur with this disease are acute renal failure, acute hepatic failure, interstitial pneumonitis, acute respiratory distress syndrome, septic shock, myocarditis, pericarditis, meningoencephalitis and also acute hearing loss is reported. ${ }^{[4,5]}$

O. tsutsugamushi targets the endothelial cells and macrophages through which it disseminates into the multiple organs via hematogenous and lymphogenous routes. It predominantly locates in the macrophages of the liver and spleen. ${ }^{[6]}$ The bacteria then cause focal or systemic vasculitis and perivasculitis in multiple organs, with various complications. The major pathophysiologic effect of rickettsial infections are: ${ }^{[7]}$

- Increased microvascular permeability due to the disruption of adherens junctions between infected endothelial cells,

- Development of interendothelial gaps,

- Formation of stress fibers and

- Change in shape of endothelial cells from polygons to large spindles.

The oxidative stress is the proposed hypothesis involved in the damage of infected endothelial cells. ${ }^{[7]}$ Though disseminated endothelial infection occurs in scrub typhus, the development of vasculitic lesions due to vaso-occlusion is rarely reported.

The Weil-Felix test (WFT), which detects antibodies produced during O. tsutsugamushi infection, is the most common and commercially available test for the diagnosis of scrub typhus in developing countries like India. The sensitivity and specificity of WFT is low and is usually positive during the $2^{\text {nd }}$ week of illness. The gold standard confirmatory tests like indirect immunoperoxidase test and the immunofluorescent assay are costly and not easily available in developing countries like India. So the diagnosis of scrub typhus is mainly by clinical suspicion and by characteristic clinical finding, eschar and serology.

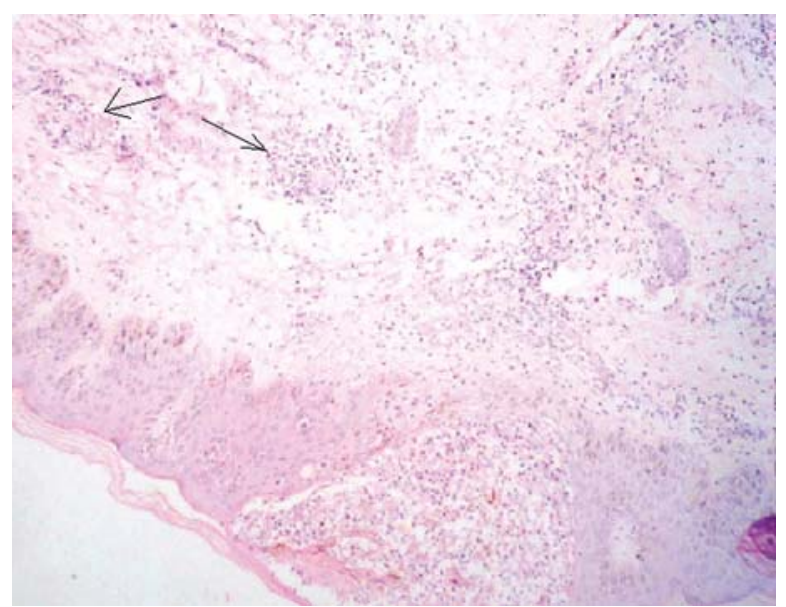

Figure 2: Normal epidermis and the dermis with inflammatory infiltrate, eosinophils and neutrophils. The blood vessels (arrows) infiltrated by inflammatory cells ( $H$ and $E, \times 200)$

The preferred drugs in the treatment include doxycycline and chloramphenicol. Doxycycline is usually given as $100 \mathrm{mg}$ PO twice daily for 7-14 days. The alternative drugs used in scrub typhus include rifampin $(600-900 \mathrm{mg} /$ day) and azithromycin. Early treatment shows better outcomes and faster resolution than delayed treatment ${ }^{[7]}$ and delayed administration of antibiotics is independently associated with major organ dysfunction. ${ }^{[8]}$ Patients treated with appropriate antibiotics typically become afebrile within 48 h of starting therapy.

Our case presented with features of vasculitis and polyarthralgia in the $1^{\text {st }}$ week of untreated illness, which is very rare and unusual. The diagnosis was confirmed by WFT. The skin biopsy revealed features suggestive of leukocytoclastic vasculitis. She responded well to treatment with doxycycline and the skin rash resolved completely in a week [Figure 1b].

In conclusion, scrub typhus can present with unusual clinical features during the course of illness and the case is reported for its rare presentation.

\section{REFERENCES}

1. Watt G, Parola P. Scrub typhus and tropical rickettsioses. Curr Opin Infect Dis 2003;16:429-36.

2. Mathai E, Rolain JM, Verghese GM, Abraham OC, Mathai D, Mathai $\mathrm{M}$, et al. Outbreak of scrub typhus in southern India during the cooler months. Ann N Y Acad Sci 2003;990:359-64.

3. Varghese GM, Abraham OC, Mathai D, Thomas K, Aaron R, Kavitha $\mathrm{ML}$, et al. Scrub typhus among hospitalised patients with febrile illness in South India: Magnitude and clinical predictors. J Infect 2006;52:56-60.

4. Cracco C, Delafosse C, Baril L, Lefort Y, Morelot C, Derenne JP, et al. Multiple organ failure complicating probable scrub typhus. Clin Infect Dis 2000;31:191-2.

5. Premaratna R, Chandrasena TG, Dassayake AS, Loftis AD, Dasch GA, de Silva HJ. Acute hearing loss due to scrub typhus: A forgotten complication of a reemerging disease. Clin Infect Dis 2006;42:e6-8.

6. Moron CG, Popov VL, Feng HM, Wear D, Walker DH. Identification of the target cells of Orientia tsutsugamushi in human cases of scrub typhus. Mod Pathol 2001;14:752-9. 
7. Valbuena G, Walker DH. Changes in the adherens junctions of human endothelial cells infected with spotted fever group Rickettsiae. Virchows Arch 2005;446:379-82.

8. Lee N, Ip M, Wong B, Lui G, Tsang OT, Lai JY, et al. Risk factors associated with life-threatening rickettsial infections. Am J Trop Med Hyg 2008;78:973-8.
How to cite this article: Praveen Kumar AS, Prasad AM, Gumpeny L, Siddappa R. Leukocytoclastic vasculitis and polyarthralgia in scrub typhus: An unusual presentation. Int J Med Public Health 2013;3:352-4.

Source of Support: Nil. Conflict of Interest: None declared. 